\title{
Test for Thermoelectric Self Cooling
}

\author{
Kuo-Chi Liu ${ }^{\text {a }}$, Yuen-Shin Chen and Mu-Kai Chen \\ Department of Mechanical Engineering, Far East University, 49 Chung Hua Rd., Hsin-Shih, Tainan 744, Taiwan
}

\begin{abstract}
Low grade waste heat is easily appears in working devices and raises their temperature. Therefore, forced convection cooling system became a necessity for keeping their performance. For saving energy, the ideal of thermoelectric self cooling that recovers and transforms the waste heat into electricity with thermoelectricity modules for driving the cooling system was proposed. In order to explore the techniques of its application, this study sets up a simulator of thermoelectric self cooling device for experimental study. The effects of the structure of heat sink on the function of thermoelectric self cooling have been investigated.
\end{abstract}

\section{Introduction}

The development of energy harvesting technology has received more attention in a variety of application areas. Currently, the environment around us is full of various waste heats which can not be recycled effectively by conventional methods. Therefore, recovering waste heat for electric power generation, especially low grade waste heat, has been regarded as the new research direction. For entire solid-state energy conversion, thermoelectric generation technology appears to have advantages in low grade waste heat recovery [1]. Thermoelectric generation technology can directly converse thermal energy into electricity by using a thermoelectric generator, which consist of p-type and n-type semiconductors, has no moving parts and is compact, quiet, highly reliable and environmentally friendly $[2,3]$.

Low grade waste heat easily appears in working devices, such as electrical power converters, transformers and control systems. Waste heat not only decreases energy efficiency, also raises working temperature of devices and reduces performance of devices. To ensure performance, forced convection cooling is always putted on devices. An extra electricity demand will be required by the cooling system. From the viewpoint of saving energy, such cooling behavior will consume more energy. Therefore, Martínez et. al. [4] proposed the idea of thermoelectric self cooling for saving electricity. The waste heat from devices was thermoelectrically converted into electricity to drive the forced cooling systems. The validity of thermoelectric self cooling has been experimentally evidenced by Martínez et. al. [4]. This paper would do an extend study to the techniques of its application. The effect of geometry of the heat sink on the dissipation efficiency is experimentally explored. It is also investigated how the rate of waste heat affects the function of generation unit.

\footnotetext{
$\overline{{ }^{a} \text { Corresponding author: kuochi.liu } @ m s a . h i n e t . n e t ~}$
}

\section{Experiment works}

\subsection{Device with thermoelectric self cooling}

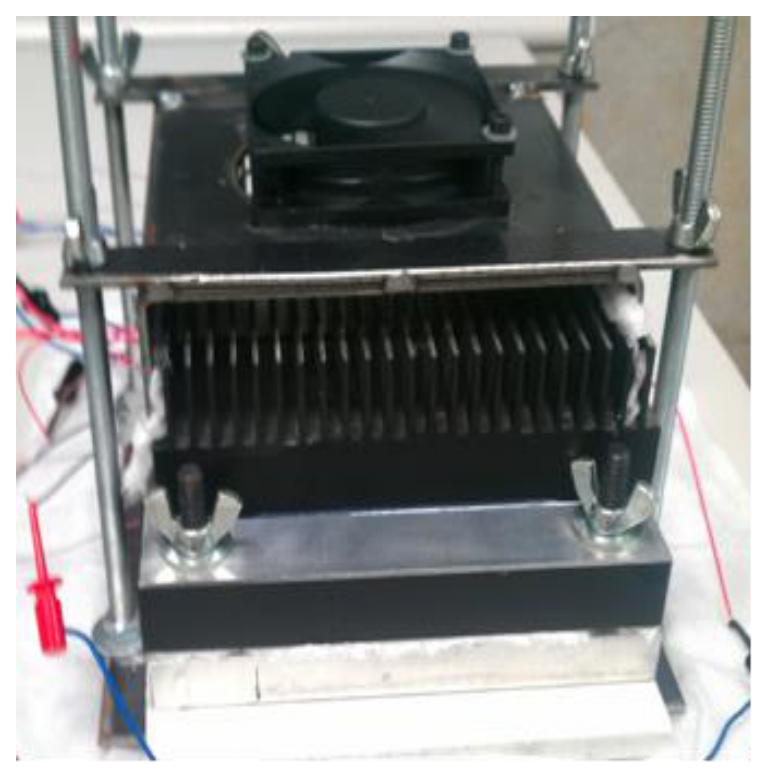

Figure 1. Photograph of the prototype made in this study.

A thermoelectric self cooling device similar to that in Ref. [4] is set up, as shown in Figure 1. A film heating resistor, which is connected in parallel to an adjustable AC power source, is sandwiched by two pieces of aluminum plate to simulate the cooled device. The power generation unit is composed of four thermoelectric modules (Kryotherm TGM-287-1.0-1.5) in series. It converts a part of the dissipation heat from the cooled device into electricity 


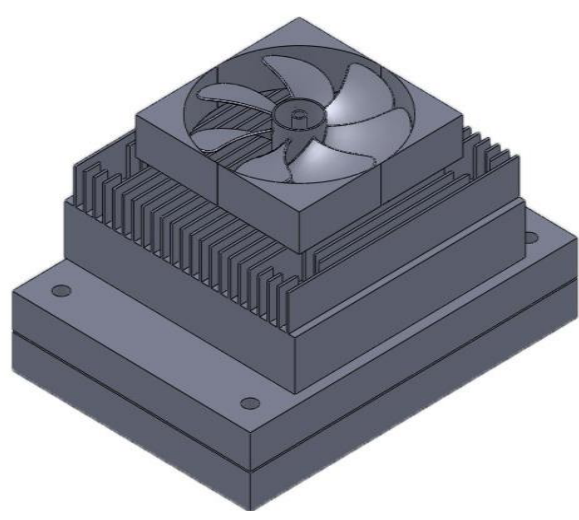

(a)

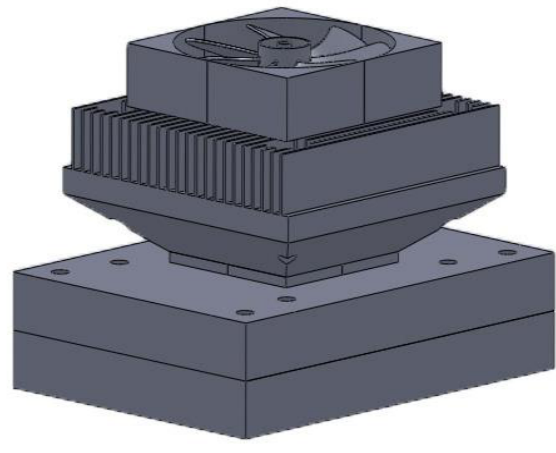

(b)

Figure 2. Perspective view of the device: (a) heat sink with cube base (situation A ) and (b) rectangular pyramidal base (situation B).

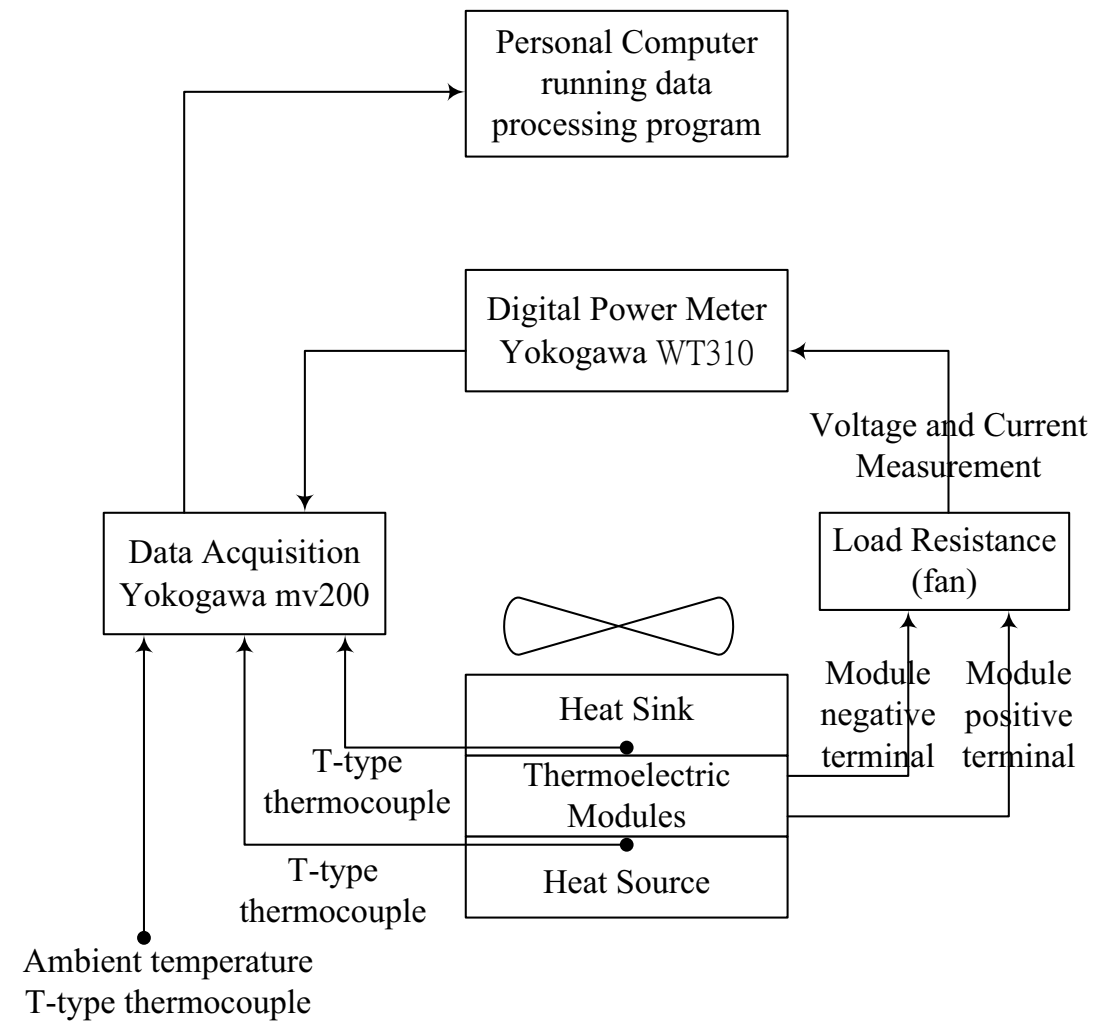

Figure 3. Scheme diagram of the test system.

to run the cooling fan, and the forced convection effect is induced. The bottom side of the cooled device is thermally insulated.

To explore the effect of heat sink, this study designs and manufactures the heat sinks with cube and rectangular pyramidal bases, as shown in Figure 2.

\subsection{Data acquisition}

This study establishes a test system for the experimental study of thermoelectric self cooling. Figure 3 shows the scheme diagram of the test system. The measurement equipments consist of a personal computer running data processing program, Yokogawa data acquisition WT310,
Yokogawa digital power meter mv200, and a number of $\mathrm{T}$ type thermal couples. Measuring the temperature of the hot and cold sides of the thermoelectric module is not easy. T-type thermocouples are attached onto two sides of the thermoelectric generation unit to measure the cold temperature, $T_{c}$, and the hot temperature, $T_{h}$.

The experiments are executed within the environments temperature $25 \pm 1{ }^{0} \mathrm{C}$. In order to explore the effect of dissipation heat, the power offered to the device is controlled by the transformer and power meter to be $80 \mathrm{~W} \cdot 120 \mathrm{~W}$ and $160 \mathrm{~W}$ respectively. The bottom side of the device is thermally insulated. Temperature, current and voltage are synchronously measured. Multiplying the voltage by the current can obtain the 
power generation rate. As a result, the effects of the geometry of heat sink and the starting delay of cooling fan on the thermoelectric generation unit can be known from the temperature difference between two sides of the generation unit and the generation power.

\section{Results and discussion}

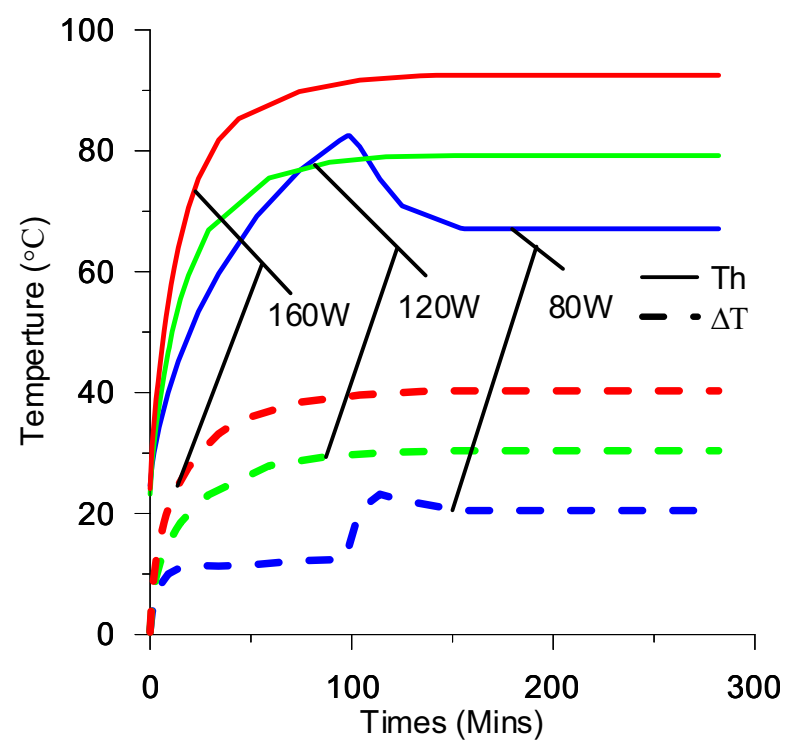

(a) For situation A.

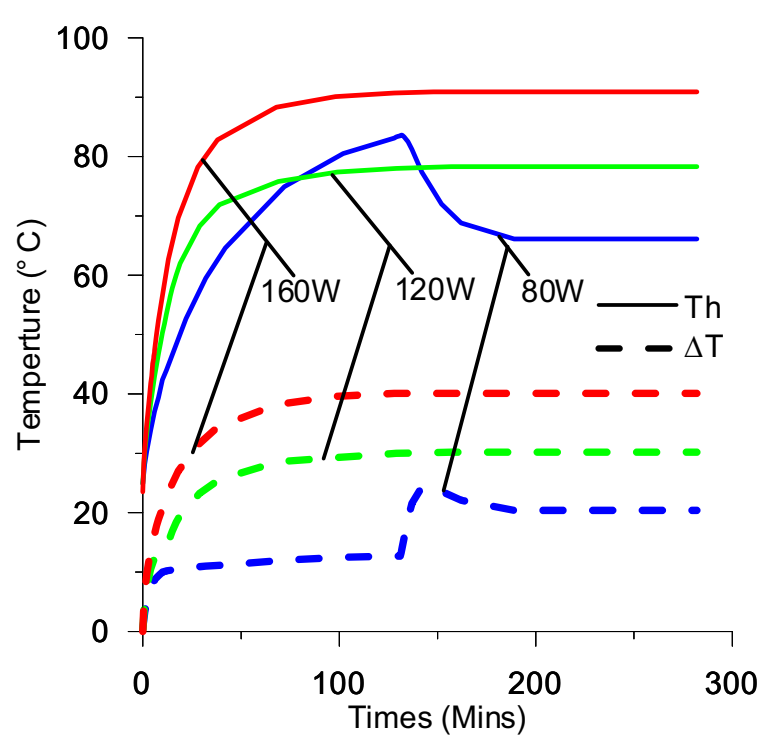

(b) For situation B.

Figure 4. Temperature difference variation between the hot and cold sides of the thermoelectric generation unit for the situations A and B.

In order to know the effect of heat sink on the performance of thermoelectric self cooling, the heat sinks, with a cube base or a rectangular pyramidal base, were used. For convenience of statement, the situation of the heat sink with a cube base and the situation of the heat sink with a rectangular pyramidal base are called as situation A and situation $\mathrm{B}$, respectively.
Figure 4 shows the temperature difference variation between the hot and cold sides of the thermoelectric generation unit for the situations $\mathrm{A}$ and $\mathrm{B}$ with the input power $80 \mathrm{~W}, 120 \mathrm{~W}$, and $160 \mathrm{~W}$. As the input power is $120 \mathrm{~W}$ and $160 \mathrm{~W}$, the temperature of hot side of the thermoelectric generation unit is rapidly raised at the beginning of heating. Therefore, the temperature difference between the hot and cold sides of the thermoelectric generation unit makes the generation unit generate an enough power to run the cooling fan. In other words, if the heat emitted from the device is enough, the cooling fan will be run with the power generated by thermoelectric modules in a short time, and the forced cooling is achieved. Relatively, as the input power is $80 \mathrm{~W}$, the difference between the hot and cold temperatures can not be enlarged and the generation power can not achieve the level that is able to run the cooling fan. Because the device is under nature convection conditions in a long time, the hot temperature continually increases, and its maximum temperature is over the maximum temperature caused by the power $120 \mathrm{~W}$, as shown in Figure 4.

It is observed from Figure 4 that the difference between the hot and cold temperatures rapidly increases for the case of the power $80 \mathrm{~W}$ after the cooling fan has been started. The hot temperature also drops along. The comparison between the experimental results of situations $\mathrm{A}$ and $\mathrm{B}$ further shows that, as the input power is $120 \mathrm{~W}$ and $160 \mathrm{~W}$, the variation of the side temperatures of situation $\mathrm{A}$ is similar to that of situation $\mathrm{B}$. The chamfered surfaces increases the dissipation area, so the hot temperature is lower in the situation B. Due to the dissipation of the chamfered surfaces, the difference between the hot and cold temperatures get a lag to achieve the level that make the thermoelectric modules generate enough power to drive the fan.

Martínez et. al. [4] considered that the phenomenon of thermal bridge may happen in the gap between the heat sink and the device and reduce the power generation. Therefore, they mounted a cold extender between the power generation unit and the heat sink in order to avoid the phenomenon of thermal bridge. The cold extender is not installed in the present experiment. The experiment results show the effect of thermal bridge is not obvious.

\section{Conclusions}

The results show that the larger the dissipation heat, the larger the difference between the hot and cold temperatures, and the effect of forced cooling more easily appears. The effect of thermal bridge is not obvious in the present study. The chamfered surfaces of heat sink with a rectangular pyramidal base do not benefit on the function of thermoelectric self cooling, but they increase the amount of heat dissipation in the early times and weaken the phenomenon of overheat.

\section{Acknowledgments}

This research was supported by the National Science Council in Taiwan through Grant NSC 101-2221-E-269006. 


\section{References}

1. L.E. Bell, Science 321, 1457 (2008).

2. J. Yang and F.R. Stabler, J. Electron Mater 38, 1245 (2009).

3. P. Yodovard, J. Khedari, and J. Hirunlabh, Energy Source 23, 213 ( 2001).

4. A. Martínez, D. Astrain, and A. Rodríguez, Energy 36, 5250 (2011). 